\title{
Dependência micorrízica do amendoinzeiro sob doses de fósforo
}

\author{
Franz Walter Rieger Hippler ( $\left.{ }^{1}\right)$; Milene Moreira $\left(^{2 *}\right)$ \\ (') Instituto Agronômico, Centro de Citricultura Sylvio Moreira, 13490-970 Cordeirópolis (SP), Brasil. \\ (2) Agência Paulista de Tecnologia dos Agronegócios, Polo Regional Centro Sul, 13400-970 Piracicaba (SP), Brasil. \\ (*) Autora correspondente: mmoreira@apta.sp.gov.br
}

Recebido: 14/jan./2013; Aceito: 22/abr./2013

\section{Resumo}

O amendoinzeiro responde positivamente quanto à produção de grãos quando associado a fungos micorrízicos arbusculares (FMA), porém é necessário verificar sua dependência para diferentes espécies em diferentes níveis de fósforo (P). Este trabaIho teve por objetivo determinar a dependência micorrízica de Arachis hypogea L. cv. Runner IAC 886 sob diferentes concentrações de P, e avaliar o desenvolvimento do hospedeiro e do endófito após a colonização. O experimento foi conduzido em casa de vegetação, em delineamento inteiramente casualizado, com duas espécies de FMA (Gigaspora rosea e Glomus clarum) e um controle não inoculado, combinados com quatro doses de P aplicados no solo: 0, 75, 150 e 250 mg kg-1. Após 50 dias da germinação do amendoim, foram avaliados a matéria seca (MS), a colonização radicular, o número de esporos no solo, a dependência micorrízica e a disponibilidade de P. Verificou-se que as plantas de amendoim apresentaram dependência micorrízica à inoculação com a espécie G. rosea nos tratamentos com baixo suprimento de P e com G. clarum somente na ausência do nutriente. A inoculação com G. rosea proporcionou maior produção de MS total na dose de $75 \mathrm{mg} \mathrm{kg}^{-1} \mathrm{de} \mathrm{P}(7,2 \mathrm{~g}$

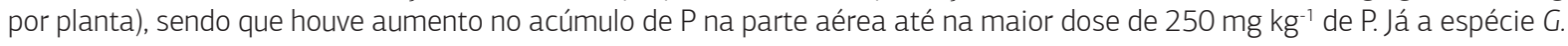
clarum apesar de ter proporcionado maiores números de esporos e colonização radicular, apresentou o menor crescimento das plantas e menor eficiência na utilização de P pela parte aérea.

Palavras-chave: fungos micorrízicos arbusculares, Arachis hypogea, adubação fosfatada.

\section{Mycorrhizal dependence of peanut plants on phosphorus levels}

\begin{abstract}
Peanut grains production responds positively when associated to arbuscular mycorrhizal fungi (AMF), but it is necessary to determine its dependence on phosphorus $(P)$ supply for in different species in plants. The aim of this study was to determine the mycorrhizal dependency of Arachis hypogea $\mathrm{L}$. to different $\mathrm{P}$ levels and to evaluate the development of host and endophyte after colonization. A greenhouse experiment was carried out in completely randomized design, with two mycorrhizal fungi species (Gigaspora rosea and Glomus clarum) and a control without the fungus, and also with different P levels applied to the soil $\left(0,75,150\right.$ and $250 \mathrm{mg} \mathrm{kg}^{-1}$ of P). After 50 days of groundnuts germination, there were evaluated dry weight (DW), root colonization, number of spores in the soil, mycorrhizal dependency and available P. The peanut plants showed mycorrhizal dependency when inoculated with $G$. rosea in low supply of $P$ and for $G$. clarum only in the absence of the nutrient. The inoculation with G. rosea produced higher DW with $75 \mathrm{mg} \mathrm{kg}^{-1}$ of $\mathrm{P}$ (7.2 g per plant), whereas there was an increase in the accumulation of $\mathrm{P}$ in shoots up to the highest dose $\left(250 \mathrm{mg} \mathrm{kg}^{-1}\right)$ of P. Although G. clarum provided higher number of spores and root colonization ratio, it had the lowest plant growth and also lower P use efficiency by shoots.
\end{abstract}

Key words: arbuscular mycorrhizal fungi, Arachis hypogea, phosphorus fertilization.

\section{INTRODUÇÃO}

As associaçóes micorrízicas com as plantas proporcionam benefícios ao crescimento das mesmas, ciclagem de nutrientes e estruturação do solo (SMith e ReAd, 2008), pois estimulam a absorção de água (JeFFries et al., 2003) e de nutrientes, especialmente aqueles de baixa mobilidade no solo, como o fósforo $(\mathrm{P})$, zinco $(\mathrm{Zn})$ e cobre (Cu) (Neumann et al., 2009). A interaçáo entre a disponibilidade de $\mathrm{P}$ no solo e o simbionte regula a eficiência da associação. Em baixas concentrações de P pode ocorrer grande colonizaçáo intrarradicular, enquanto em níveis elevados de $\mathrm{P}$ a colonização tende a diminuir (CARdoso et al., 2003).

Mesmo não ocorrendo especificidade entre fungos micorrízicos arbusculares (FMA) e plantas hospedeiras, trabalhos demonstraram que Glomus clarum destacou-se em relação a outros FMA em plantas cultivadas em diferentes concentraçóes de P no solo, como algodoeiro (SiqueIra et al., 1986), trevo vesiculoso 
(Pessoa et al., 1997), soja (Nogueira e Cardoso, 2000) e cedro (Rocha et al., 2006).

A dependência micorrízica é definida pela dependência que a planta colonizada por FMA tem para atingir seu maior crescimento e/ou produção em um determinado nível de fertilidade do solo (Moreira e Siqueira, 2006). Os FMA podem beneficiar a cultura do amendoim, permitindo a redução do uso de adubos e dos custos de produção. Assim, é importante estudar diferentes espécies de FMA em uma mesma planta, para selecionar FMA eficientes em promover o crescimento de seu hospedeiro (Paula et al., 1990; Rocha et al., 2006).

O cultivo comercial do amendoim ocorre muitas vezes em solos empobrecidos, já que geralmente é realizado em áreas de reforma de canaviais e pastagens degradadas. A introdução desta leguminosa no sistema pode ajudar na recuperação destes solos, capaz de nodular e fixar nitrogênio em simbiose com grande variedade de bactérias do gênero Rhizobium, além de ser uma espécie micotrófica e poder se associar com FMA, proporcionando desta forma benefícios ao sistema de produção e ao meio ambiente pela incorporação de nitrogênio ou pela capacidade de aumentar os propágulos micorrízicos no solo (Miranda, 2008). Entretanto, cada planta apresenta peculiaridades quando em associação com FMA, o que vai depender da espécie de FMA associada, do ambiente e da eficiência da simbiose.

O presente trabalho teve por objetivo avaliar a resposta do amendoinzeiro à colonização com duas espécies de FMA e determinar o grau de dependência micorrízica em diferentes doses de $\mathrm{P}$ aplicadas ao solo.

\section{MATERIAL E MÉTODOS}

O experimento foi realizado em casa de vegetação, com delineamento inteiramente casualizado, em esquema fatorial 3x4, com duas espécies de FMA (Gigaspora rosea e Glomus clarum) e um controle sem FMA, combinadas com quatro doses de $\mathrm{P}\left(0,75,150\right.$ e $\left.250 \mathrm{mg} \mathrm{kg}^{-1}\right)$, com cinco repetiçôes por tratamento, num total de 60 vasos de $3 \mathrm{~L}$ cada, preenchidos com solo.

O solo utilizado foi proveniente de uma mistura de Neossolo Quartzarênico (EMBRAPA, 2006) com uma parte de areia de rio lavada $(2: 1 ; \mathrm{v}: \mathrm{v})$. Após autoclavagem do substrato por duas horas a $121^{\circ} \mathrm{C}$, coletou-se uma amostra para a realização da análise química, cujos resultados foram: $\mathrm{pH}\left(\mathrm{CaCl}_{2}\right) 4,3 ; \mathrm{P}$ (resina) $=6,0 \mathrm{mg} \mathrm{dm}^{-3} ; \mathrm{Ca}=9,0 ; \mathrm{Mg}=2,0$, $\mathrm{Al}=3,0$ e $\mathrm{K}=0,6 \mathrm{em} \mathrm{mmol} \mathrm{dm}^{-3}$. Realizou-se a calagem no solo (QuagGio e Godor, 1996) para elevar a saturação por bases $(\mathrm{V})$ a $60 \%$, incubando-se porçôes de $10 \mathrm{~kg}$, previamente umedecidas e acondicionadas em sacos plásticos, por um período de 20 dias à temperatura ambiente. Posteriormente, adicionou-se o $\mathrm{P}$ nas doses de 75, $150 \mathrm{e}$ $250 \mathrm{mg} \mathrm{kg}^{-1}$ na forma de $\mathrm{KH}_{2} \mathrm{PO}_{4}$ P.A. A mistura solo-areia foi inserida nos vasos previamente lavados e desinfestados com hipoclorito de sódio a 5\% (v/v).

O substrato dos vasos foi infestado com os propágulos de FMA ( $G$. clarum e G. rosea), utilizando-se como inóculo $20 \mathrm{~g}$ de solo contendo propágulos do fungo obtidos em vasos de multiplicação com Brachiaria decumbens. $\mathrm{O}$ inóculo foi introduzido durante o transplante do amendoim (Arachis hypogea) cultivar Runner IAC 886. Nos vasos controle, foram utilizadas as mesmas quantidades de solo rizosférico e raízes de plantas de braquiária não micorrizadas.

As sementes de amendoim foram desinfetadas em solução de hipoclorito de sódio a $0,5 \%(\mathrm{v} / \mathrm{v})$ durante 5 minutos, lavadas com água esterilizada e colocadas em um pré-germinador por 4 dias. Após a germinação, 3 sementes pré-germinadas foram transplantadas por vaso e, 20 dias após o transplante, foi realizado o desbaste, deixando uma planta por vaso. Aos 20 dias após o plantio, adicionaram-se $30 \mathrm{~mL}$ da soluçáo de Hoagland isenta de P. As plantas foram irrigadas periodicamente com água destilada conforme a necessidade.

No florescimento, as plantas foram colhidas, separadas em parte aérea e raiz e lavadas. Após secagem em estufa a $60^{\circ} \mathrm{C}$ por 48 horas determinou-se a matéria seca da parte área (MSPA) e de raiz (MSR). O somatório da MSPA e MSR determinou a matéria seca total (MStotal). A partir da MStotal, foi determinada a dependência micorrízica (DM) de acordo com o seguinte cálculo: matéria seca de plantas micorrizadas menos matéria seca de plantas não micorrizadas, dividido por matéria seca de plantas micorrizadas e multiplicado por 100 (Plenchette et al., 1983). Após a secagem e a pesagem, a parte aérea das plantas foi moída e o teor de P quantificado segundo o método de Malavolta et al. (1989). O P acumulado $\left(\mathrm{P}_{\mathrm{PA}}\right)$ foi obtido a partir do produto entre o teor de $\mathrm{P}$ na parte aérea e a MSPA. A partir destes valores, calculou-se a eficiência de utilização de $\mathrm{P}$ na parte aérea $\left(\right.$ EUP, $\left.\mathrm{g}^{2} \mathrm{mg}^{-1}\right)=(\mathrm{MSPA}, \mathrm{g})^{2} /($ conteúdo de $\mathrm{P}$ na parte aérea, mg) (Siddiqi e Glass, 1981).

Determinou-se o número de esporos de FMA pelo método de peneiramento por via úmida em uma alíquota de $50 \mathrm{~g}$ de solo, seguindo-se centrifugação a $700 \mathrm{xg}$ por 3 minutos em sacarose $(40 \%, \mathrm{~m} / \mathrm{v})$ (Jenkins, 1964). As quantificaçóes dos esporos foram feitas sob microscópio estereoscópico com aumento de 40 vezes, em placas com canaletas concêntricas (Gerdemann e Nicolson, 1963). Para determinação da colonização micorrízica, retiraram-se as raízes finas, que foram lavadas, clarificadas com $\mathrm{KOH}(10 \%$; v/v) e coradas com azul de tripano em lactoglicerol $(0,05 \% ; \mathrm{v} / \mathrm{v})$. A determinação da colonizaçáo radicular foi realizada pela técnica de interseção dos quadrantes (Phillips e Hayman, 1970; Giovanetti e Mosse, 1980). As raízes das plantas não inoculadas com FMA foram analisadas e apresentaram-se isentas de colonização radicular. 
Para normalização da distribuição, os dados de colonização radicular foram transformados para arcsen $(\mathrm{x} / 100)^{1 / 2}$ e o número de esporos para $(\mathrm{x}+0,5)^{1 / 2}$. As variáveis foram submetidas à análise de variância, desdobrando-se as interaçôes. Aplicou-se o teste de Tukey a 5\% de significância para a comparação das médias do fator qualitativo e a análise de regressão para o fator quantitativo.

\section{RESULTADOS E DISCUSSÃO}

A inoculaçáo com $G$. rosea e a adiçáo de $\mathrm{P}$, até a dose de $150 \mathrm{mg} \mathrm{kg}^{-1}$, promoveram o crescimento das plantas de amendoim, com base nos incrementos da MSPA e MStotal $(\mathrm{p}<0,01)$ (Figuras 1 e 2$)$, no qual foram obtidos os maiores valores (4,9 e 7,2 g por planta) quando comparados com plantas inoculadas com G. clarum e plantas controle. Verificou-se que o ponto máximo de desenvolvimentos da MSPA foi na concentraçáo de $167 \mathrm{mg} \mathrm{kg}^{-1}$ de $\mathrm{P}$ e para a MStotal na de $132 \mathrm{mg} \mathrm{kg}^{-1}$ de $\mathrm{P}$, sendo que para concentrações maiores de P houve redução da MSPA e MStotal na presença desse endófito. Efeito semelhante foi verificado em araucária (Moreira-Souza e Cardoso, 2002), que quando inoculadas com $G$. rosea apresentaram maior desenvolvimento em condiçốes de baixas concentraçôes de P. Apesar de essa espécie de FMA ter proporcionado maior crescimento no amendoinzeiro, outros estudos demonstraram ser efetiva para o desenvolvimento de mudas de plantas perenes (ANTUNES et al., 1988). Em culturas anuais como soja e Stylosanthes guianensis a inoculação com Gigaspora spp. não mostrou-se eficiente (Lambais e Cardoso, 1990; Nogueira e Cardoso, 2000).

As plantas inoculadas com $G$. clarum demonstraram resposta de menor intensidade quanto à produção de MSPA e MStotal, 2,5 e 3,1 g por planta, respectivamente, quando comparadas com plantas inoculadas com G. rosea e não inoculadas. Houve acréscimos de MSPA e MStotal para as doses de 130 e $150 \mathrm{mg} \mathrm{kg}^{-1}$ de $\mathrm{P}$, respectivamente, e decréscimos entre estes valores e $250 \mathrm{mg} \mathrm{kg}^{-1}$ de $\mathrm{P}$. As plantas não colonizadas (controle) responderam às doses crescentes de $\mathrm{P}$, sendo que aquelas maiores (150 e $250 \mathrm{mg} \mathrm{kg}^{-1}$ de P) proporcionaram maior MSR, ou seja, cerca de $2,0 \mathrm{~g} \mathrm{~kg}^{-1}$ de P. Neste tratamento, o ponto de máximo desenvolvimento foi na dose $181 \mathrm{mg} \mathrm{kg}^{-1}$ de $\mathrm{P}$ para a MSPA e $126 \mathrm{mg} \mathrm{kg}^{-1}$ de P para a MStotal.

$\mathrm{O}$ teor de $\mathrm{P}$ na parte aérea aumentou com o acréscimo das doses de $\mathrm{P}$ no solo dentro do mesmo tratamento, mas não houve diferenças entre os diferentes FMA inoculados (dados não apresentados). Porém, o efeito positivo da inoculação de FMA na produção de MSPA proporcionou maior acúmulo de $\mathrm{P}\left(\mathrm{P}_{\mathrm{PA}}\right)$ na parte aérea de plantas colonizadas com $G$. rosea que receberam as duas maiores doses de $\mathrm{P}$, com aproximadamente $1,0 \mathrm{mg}$ por planta $(\mathrm{p}<0,05)$ (Figura 3). Já as plantas colonizadas com G. clarum e as plantas sem inóculo não mostraram aumento significativo. Ao contrário do verificado no presente estudo, a espécie G. clarum foi eficiente em promover o crescimento e absorção de P em mudas de cedro (Rocha et al., 2006).

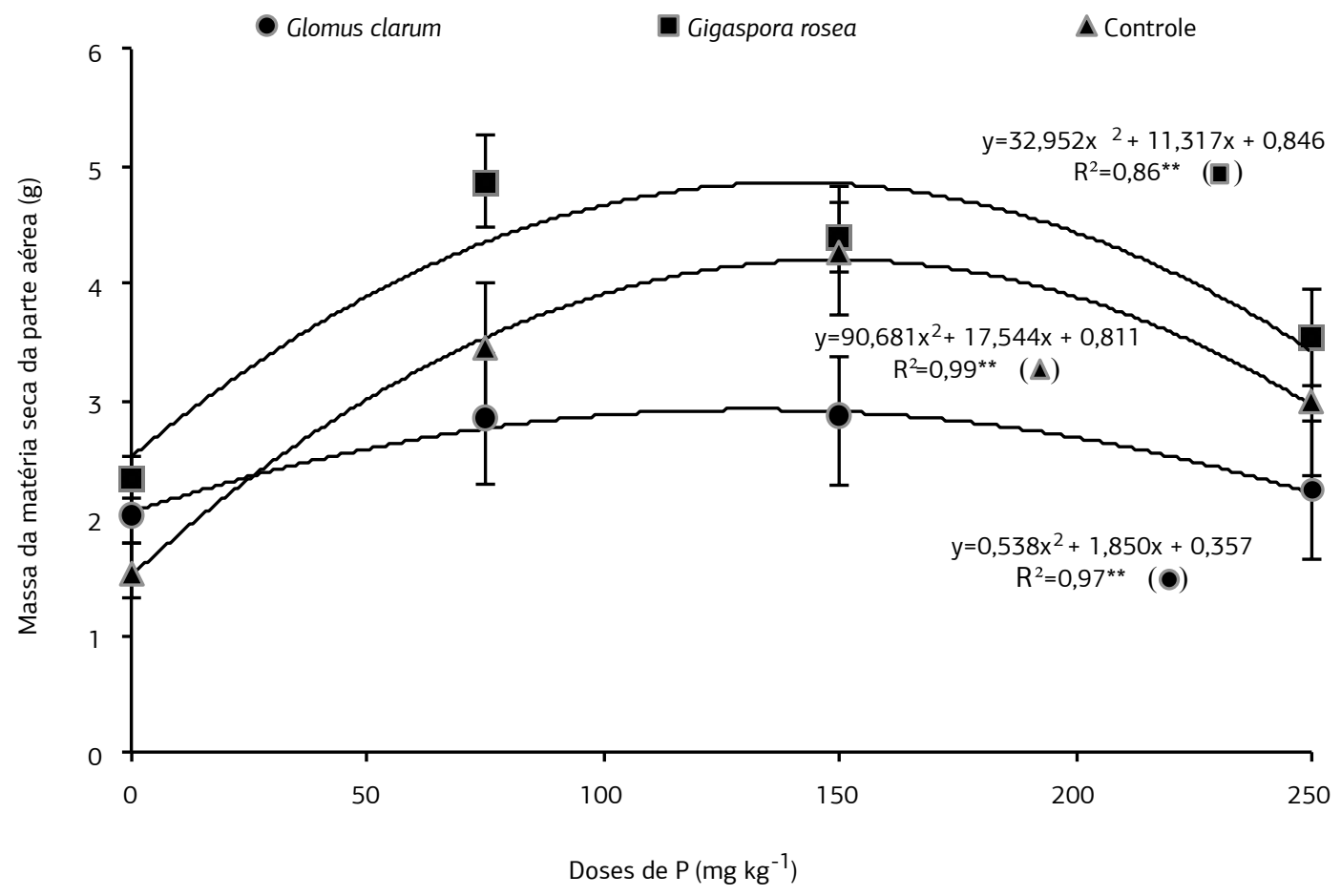

Figura 1. Matéria seca da parte aérea de plantas de Arachis hypogea colonizadas com fungos micorrízicos arbusculares (Glomus clarum e Gigaspora rosea) e não colonizadas (controle), aos 50 dias após o plantio, considerando doses fósforo. Barra vertical em cada ponto representa o desvio-padrão da média. ${ }^{* *} \mathrm{p}<0,01$. 


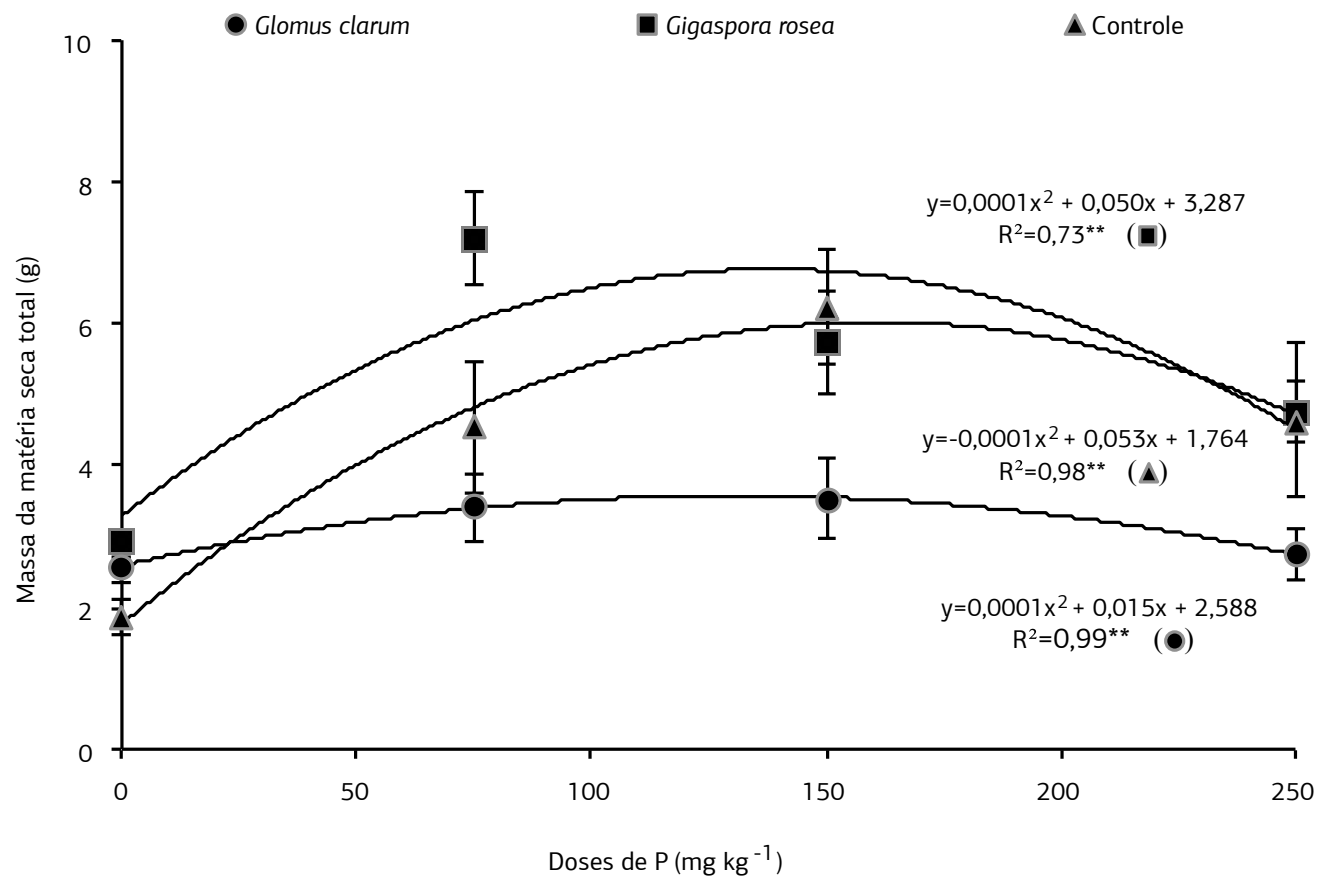

Figura 2. Matéria seca total de plantas de Arachis hypogea colonizadas com fungos micorrízicos arbusculares (Glomus clarum e Gigaspora rosea) e não colonizadas (controle), aos 50 dias após o plantio, considerando doses fósforo. Barra vertical em cada ponto representa $o$ desvio-padrão da média. ${ }^{* *} \mathrm{p}<0,01$.

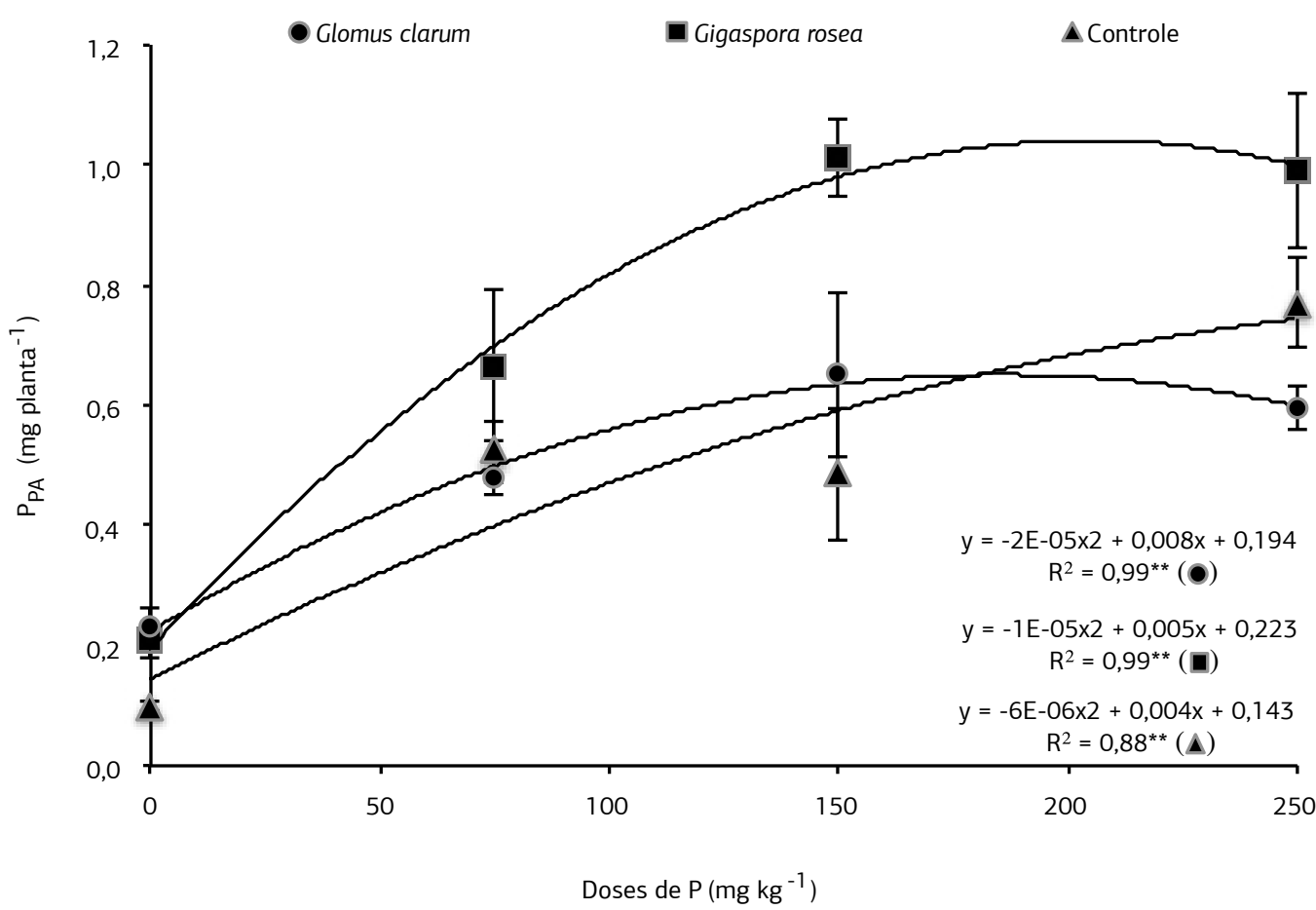

Figura 3. Acúmulo de fósforo na parte aérea $\left(\mathrm{P}_{\mathrm{PA}}\right)$ de plantas de Arachis hypogea colonizadas com fungos micorrízicos arbusculares (Glomus clarum e Gigaspora rosea) e não colonizadas (controle), aos 50 dias após o plantio, considerando doses fósforo. Barra vertical em cada ponto representa o desvio-padrão da média. ${ }^{* *} \mathrm{p}<0,01$.

Ainda, Nogueira e Cardoso (2000) verificaram que plantas de soja responderam à inoculação com FMA do gênero Glomus, mas náo com Gigaspora, demonstrando a importância de se verificar a compatibilidade funcional entre FMA e planta hospedeira. Esse gênero de FMA, embora muito encontrado na rizosfera do amendoinzeiro em condiçóes de campo, não foi eficiente em promover o desenvolvimento das plantas (Hippler e Moreira, 2010). 
Apesar do incremento na absorção de $\mathrm{P}$ pelas raízes associadas ao fungo ser atribuído ao aumento da superfície de absorção (Hayman e Mosse, 1972), o sistema com micorriza não depende exclusivamente de uma exploração física, mas principalmente da presença e da expansão de sítios de absorçáo de maior afinidade por $\mathrm{P}$ nas raízes (Cress et al., 1979), como verificado nas plantas colonizadas com $G$. rosea. Ainda, plantas micorrizadas tendem a apresentar maior velocidade máxima de absorção $\left(\mathrm{V}_{\max }\right)$ de $\mathrm{P}$ quando comparadas a plantas não micorrizadas (FAQUIN et al., 1990). Em plantas de feijão, Silveira e Cardoso (2004) verificaram maior $\mathrm{V}_{\text {max }}$ de absorção de $\mathrm{P}$ em plantas inoculadas com Glomus etunicatum no momento do florescimento, porém sendo semelhante a plantas não micorrizadas na fase de enchimento de gráos. Assim, o maior acúmulo de P na parte aérea das plantas colonizadas por $G$. rosea pode ser em virtude desta espécie ter proporcionado maiores quantidades de sítios de absorção de $\mathrm{P}$, pelas hifas (Silveira e Cardoso, 2004), e assim maior $\mathrm{V}_{\text {max }}$ até o momento avaliado que foi o do florescimento.

Quando verificada a eficiência na utilização do fósforo (EUP) na parte aérea, apesar de não haver interação entre os tratamentos de inoculaçáo de FMA e de doses de $\mathrm{P}$, as plantas de amendoim obtiveram baixa EUP quando inoculadas com G. clarum $\left(14,0 \mathrm{~g}^{2} \mathrm{mg}^{-1}\right)$, e quando cultivadas na maior dose de $\mathrm{P}\left(10,7 \mathrm{~g}^{2} \mathrm{mg}^{-1}\right)$ (Tabela 1$)$. Esta menor eficiência para os tratamentos com as maiores de doses de P ocorreu devido ao menor crescimento das plantas (MStotal) em relação a quantidade de $\mathrm{P}$ absorvido e acumulado no tecido vegetal, quando houve maior disponibilidade do nutriente (MarsCHNER, 1995), confirmando assim o verificado nas culturas da soja e do feijão (Procópio, 2005). Plantas expostas a altas concentraçóes de P tendem a armazená-lo no vacúolo na forma inorgânica, contribuindo para aumentar o teor do nutriente na planta, porém sem participar diretamente de reaçôes metabólicas ou na formação de estruturas (FurTini NeTO et al., 1998; Zambrosi et al., 2012).

A ocorrência do menor crescimento de diversas espécies de plantas, quando micorrizadas e cultivadas em alta disponibilidade de P, é caracterizada como

Tabela 1. Eficiência na utilização de fósforo $\left(\mathrm{g}^{2} \mathrm{mg}^{-1}\right)$ na parte aérea de plantas de Arachis hypogea colonizadas com fungos micorrízicos arbusculares (Glomus clarum e Gigaspora rosea) e não colonizadas (controle), cultivadas sob diferentes doses de fósforo

\begin{tabular}{lccccc} 
Tratamentos $^{(1)}$ & \multicolumn{5}{c}{ Doses de fósforo $\left(\mathbf{m g ~ k g}^{-1}\right)$} \\
\cline { 2 - 6 } & $\mathbf{0}$ & $\mathbf{7 5}$ & $\mathbf{1 5 0}$ & $\mathbf{2 5 0}$ & Média \\
\hline Controle & 23.7 & 22.8 & 37.8 & 11.2 & $23.9^{\mathrm{a}}$ \\
\hline $\begin{array}{l}\text { Glomus clarum } \\
\text { Gigaspora }\end{array}$ & 17.8 & 17.4 & 12.3 & 8.4 & $14.0^{\mathrm{b}}$ \\
$\begin{array}{l}\text { rosea } \\
\text { Média }\end{array}$ & 26.5 & 35.6 & 19.1 & 12.6 & $23.5^{\mathrm{a}}$ \\
\hline
\end{tabular}

Não houve interação significativa entre fungos micorrízicos arbusculares e doses de fósforo. As médias acompanhadas por letras minúsculas iguais na mesma coluna e por letras maiúsculas na mesma linha não diferem pelo teste de Tukey a $5 \%$. depressão de crescimento, e que envolve diversos fatores, principalmente bioquímicos (SENA et al., 2004). Esta diminuição na biomassa pode ocorrer devido ao aumento da colonização interna, de estruturas ativas do fungo no sistema radicular, assim apesar de haver o dreno de carboidratos ao fungo, não há retorno, por parte do fungo, no fornecimento de nutrientes e outros compostos (SENA et al., 2004), resultando na queda de produção de biomassa e crescimento.

Em relação ao número de esporos, as plantas inoculadas com G. clarum apresentaram cerca de 90 e 130 esporos em $50 \mathrm{~g}$ de solo seco, respectivamente nas doses de 75 e $250 \mathrm{mg} \mathrm{kg}^{-1}$ de P. Plantas inoculadas com G. rosea apresentaram valores menores, sendo 40 esporos em $50 \mathrm{~g}$ de solo seco, o maior valor encontrado nas plantas que não receberam $\mathrm{P}$ (Figura 4).

As percentagens de colonização radicular em plantas colonizadas com G. clarum verificadas na ausência de aplicação de $\mathrm{P}$ e até a dose $150 \mathrm{mg} \mathrm{kg}^{-1} \mathrm{de} P$ variaram entre 67 a $79 \%$ e na maior dose de $\mathrm{P}\left(250 \mathrm{mg} \mathrm{kg}^{-1} \mathrm{de} \mathrm{P}\right)$, as raízes apresentaram colonização de 30\% (Figura 5). Já as plantas inoculadas com $G$. rosea apresentaram entre 9 a 19\% de colonização radicular. Mesmo apresentando maior colonização radicular, o tratamento inoculado com $G$. clarum produziu MStotal inferior aos demais tratamentos, sendo que estas produziram 30\% menos de MStotal quando comparadas as plantas não colonizadas.

A percentagem de colonização elevada pode diminuir o desenvolvimento radicular, e isso pode ocorrer quando o hospedeiro entra em fase reprodutiva (Allen, 2001), como foi o caso do G. clarum no presente estudo, em que se verificou a presença de muitos esporos (até 130 esporos por $50 \mathrm{~g}$ de solo) (Figura 4) nas raízes e também a maior esporulação no solo e menor desenvolvimento das plantas. $\mathrm{Na}$ fase do florescimento e após, ocorre um desvio do dreno de carboidratos para

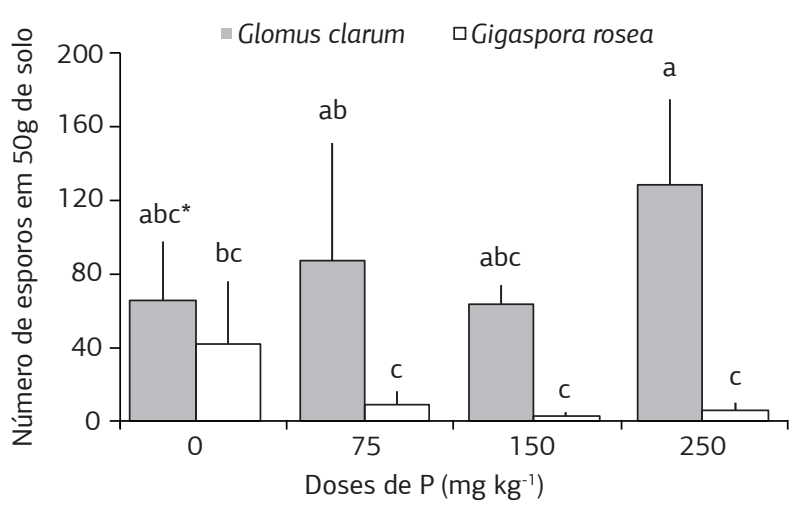

Figura 4. Número de esporos em plantas de Arachis hypogea colonizadas com fungos micorrízicos arbusculares Glomus clarum e Gigaspora rosea no período do florescimento, em diferentes doses de fósforo. ${ }^{*}$ Médias seguidas pela mesma letra, não diferem significativamente pelo teste de Tukey $(\mathrm{p}<0,05)$. Barra vertical em cada coluna representa o desvio-padrão da média. 
o desenvolvimento das estruturas reprodutivas, flores, vagens e frutos, em detrimento ao desviado aos FMA (CARdoso et al., 2003).

Plantas inoculadas com $G$. rosea apresentaram os maiores índices de dependência micorrízica, entre 40 e $37 \%$ nas menores concentraçóes de P $\left(0\right.$ e $75 \mathrm{mg} \mathrm{kg}^{-1}$ de $\mathrm{P}$, respectivamente), dentre todos os tratamentos, enquanto as plantas inoculadas com G. clarum apresentaram dependência micorrízica de $27 \%$ na dose $0 \mathrm{mg} \mathrm{kg}^{-1}$ de $\mathrm{P}$ (Figura 6). Essa dependência ocorre também em relação a inoculação do amendoinzeiro com FMA nativos em diferentes doses de P, neste caso, plantas inoculadas com G. rosea, sendo que apresentam a maior dependência micorrízica quando supridas com $60 \mathrm{mg} \mathrm{kg}^{-1}$ de $\mathrm{P}$ (Hippler et al., 2011). Nas maiores doses de P (150 e $250 \mathrm{mg} \mathrm{kg}^{-1} \mathrm{de}$ P) o amendoinzeiro não apresentou dependência positiva para nenhum dos endófitos inoculados. A alta disponibilidade de nutrientes, especialmente de P, como encontrado no presente estudo em duas maiores doses, diminuem a colonização micorrízica nas

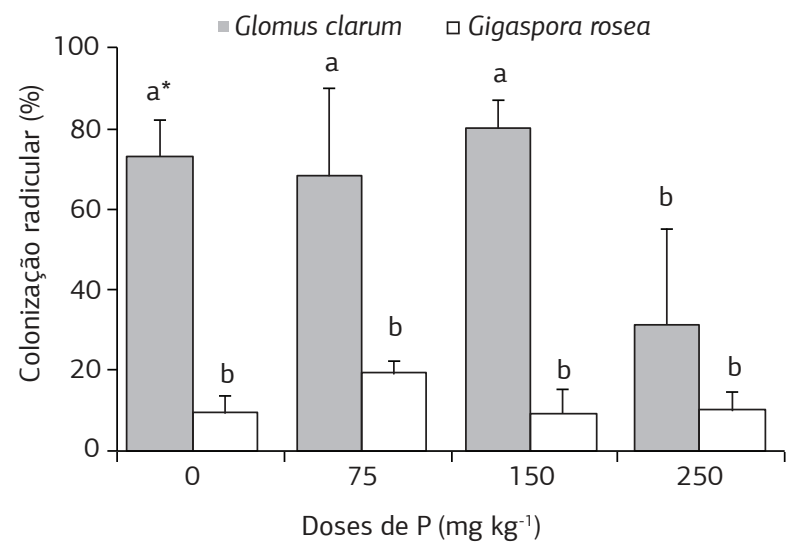

Figura 5. Colonização radicular em plantas de Arachis hypogea colonizadas com fungos micorrízicos arbusculares Glomus clarum e Gigaspora rosea no período do florescimento, em diferentes doses de fósforo. ${ }^{*}$ Médias seguidas pela mesma letra, não diferem significativamente pelo teste de Tukey $(p<0,05)$. Barra vertical em cada coluna representa o desvio-padrão da média.

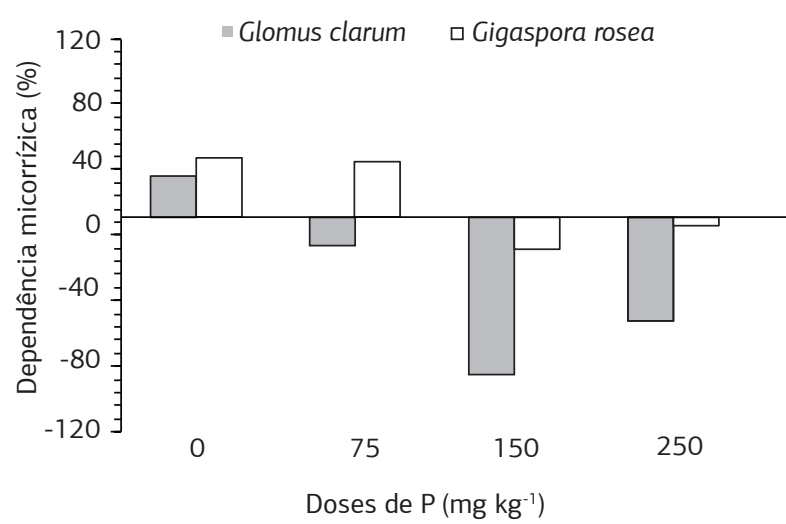

Figura 6. Dependência e micorrízica na produção de matéria seca de plantas micorrizadas de Arachis hypogea sob diferentes doses de fósforo. diversas espécies, devido a menor dependência da planta pelo FMA (Melloni e Cardoso, 1999; Nogueira e Cardoso, 2000; Moreira-Souza e Cardoso, 2002).

Assim, apesar do amendoinzeiro apresentar dependência micorrízica para ambas as espécies, demonstra-se a eficiência em utilização de $\mathrm{P}$ pelo amendoinzeiro colonizado com G. rosea, já que esta combinação proporcionou maior produção de biomassa enquanto as plantas não inoculadas tiveram o máximo de produção na dose de $126 \mathrm{mg} \mathrm{kg}^{-1}$ de $\mathrm{P}$.

\section{CONCLUSÃO}

A espécie $G$. Clarum apresentou maior esporulaçáo e maior grau de colonização radicular do que G. rosea, a interação não foi eficiente em promover maior crescimento das plantas de amendoim, além de ter apresentado menor eficiência na utilizaçáo de P pela parte aérea. Por outro lado, a inoculação com G. rosea proporcionou maior produção de biomassa somente na dose de $75 \mathrm{mg} \mathrm{kg}^{-1}$ de P. Essa associação aumentou o acúmulo de $\mathrm{P}$ na parte aérea inclusive na maior dose de $\mathrm{P}$ adicionada ao solo. A concentraçáo de $\mathrm{P}$ no solo influenciou o desenvolvimento das plantas independentemente da inoculação com FMA, sendo as maiores dependências micorrízicas verificadas quando inoculadas com a espécie $G$. rosea, especialmente nas doses de até $75 \mathrm{mg} \mathrm{kg}^{-1}$ de $\mathrm{P}$.

\section{AGRADECIMENTOS}

A Fundação de Amparo a Pesquisa do Estado de Sáo Paulo (FAPESP) pelo auxílio financeiro (2006/03660-8) e pela bolsa de iniciação científica (2008/56785-8) concedida ao primeiro autor. Os autores agradecem também à Sra. Maria Alice Mascarenhas pelo auxílio técnico.

\section{REFERÊNCIAS}

ALLEN, M.F. Modeling arbuscular mycorrizal infection: is \% infection an appropriate variable? Mycorrhiza, v.10, p.255-258, 2001. DOI: $10.1007 / \mathrm{s} 005720000081$

ANTUNES, V.; SILVEIRA, A.P.; CARDOSO, E.J.B.N. Interaçâo entre diferentes tipos de solo e fungos micorrízicos vesículoarbusculares na produção de mudas de café (Coffea arabica L.). Turrialba, v.38, p.117-122, 1988.

CARDOSO, E.J.B.N.; NAVARRO, R.B.; NOGUEIRA, M.A. Absorção e translocação de manganês por plantas de soja micorrizadas, sob doses crescentes deste nutriente. Revista Brasileira de Ciência do Solo, v.27, p.415-423, 2003. DOI: 10.1590/S010006832003000300003 
CRESS, W.A.; THRONEBERRY, G.O.; LINDSEY, D.L. Kinetics of phosphorus absorption by mycorrhizal and non mycorrhizal tomato roots. Plant Physiology, v.64, p.484-487, 1979. DOI: 10.1104/pp.64.3.484

EMPRESA BRASILEIRA DE PESQUISA AGROPECUÁRIA (EMBRAPA). Centro Nacional e Pesquisa em Solos. Sistema Brasileiro de Classificação de Solos. Brasília: EMBRAPA/SPI; Rio de Janeiro: EMBRAPA Solos, 2006. 306p.

FAQUIN, V.; MALAVOLTA, E.; MURAOKA, T. Cinética da absorção de fosfato em soja sob influência de micorriza visículo-arbuscular. Revista Brasileira de Ciência do Solo, v.14, p.41-48, 1990 .

FURTINI NETO, A.E.; BARROS, N.F.; NOVAIS, R.F.; OLIVEIRA, M.F.G. Fraçóes fosfatadas em mudas de Eucalyptus. Revista Brasileira de Ciência do Solo, v.22, p.267-274, 1998.

GERDEMANN, J.W.; NICOLSON, T.H. Spores of mycorrhizal Endogone species extracted from soil by sieving and decanting. Transactions of the British Mycological Society, v.46, p.235246, 1963. DOI: 10.1016/S0007-1536(63)80079-0

GIOVANETTI, M.; MOSSE, B. An evaluation of techniques for measuring vesicular arbuscular mycorrhizal infection in roots. New Phytologist, v.84, p.489-500, 1980. DOI: 10.1111/j.14698137.1980.tb04556.x

HAYMAN, D.S., MOSSE, B. The role of vesicular-arbuscular mycorrhizal in the removal of phosphorus from soil by plant roots. Review in Ecology and Biology of Soil, v.9, p.483-470, 1972.

HIPPLER, F.W.R.; MOREIRA, M.; DIAS, N.M.S.; HERMANN, E.R. Fungos micorrízicos arbusculares nativos e doses de fósforo no desenvolvimento do amendoim RUNNER IAC 886. Revista Ciência Agronômica, v.42, p.605-610, 2011. DOI: 10.1590/ S1806-66902011000300005

HIPPLER, F.W.R.; MOREIRA, M. Plantas de amendoim inoculadas com Bradyrhizobium e fungos micorrízicos arbusculares. Ecossistema, v.34-35, p.93-97, 2010.

JEFFRIES, P.; GIANINAZZI, S.; PEROTTO, S.; TURNAU, K.; BAREA, J.M. The contribution of arbuscular mycorrhizal fungi in sustainable maintenance of plant health and soil fertility. Biology and Fertility of Soils, v.37, p.1-16, 2003. DOI: 10.1007/s00374002-0546-5

JENKINS, W.R. A rapid centrifugal-flotation technique for separating nematodes from soil. Plant Disease Report, v.48, p.692, 1964.

LAMBAIS, M.R.; CARDOSO, E.J.B.N. Response of Shylosanthes huianensis to endomycorrhizical fungi inoculation as affected by lime and phosphorus applications. Plant and Soil, v.129, p.283289, 1990. DOI: $10.1007 / \mathrm{BF} 00032424$

MALAVOLTA, E.; VITTI, G.C.; OLIVEIRA, S.A. Avaliação do estado nutricional das plantas: princípios e aplicaçóes. Piracicaba: Associação Brasileira para Pesquisa da Potassa e do Fosfato, 1989. 201p.
MARSCHNER, H. Mineral nutrition of higher plants. London: Academic Press, 1995. 889p.

MELLONI, R.; CARDOSO, E.J.B.N. Quantificação de micélio extra-radicular de fungos micorrízicos arbusculares em plantas cítricas: II., comparação entre diferentes espécies cítricas e endófitos. Revista Brasileira de Ciência do Solo, v.23, p.59-67, 1999.

MIRANDA, J.C.C. Cerrado: micorriza arbuscular, ocorrência e manejo. Planaltina: Embrapa, 2008. 169p.

MOREIRA-SOUZA, M.; CARDOSO, E.J.B.N. Dependência micorrízica de Araucaria angustifolia (Bert.) O. KTZE. sob doses de fósforo. Revista Brasileira de Ciência do Solo, v.26, p.905$912,2002$.

MOREIRA, F.M.S.; SIQUEIRA, J.O. Microbiologia e bioquímica do solo. Lavras: Editora UFLA, 2006. 729p.

NEUMANN, E.; SHMID, B.; RÖMHELD, V.; GEORGE, E. Extraradical development and contribution to plant performance of an arbuscular mycorrhizal symbiosis exposed to complete or partial rootzone drying. Mycorrhiza, v.20, p.13-23, 2009. DOI: $10.1007 / \mathrm{s} 00572-009-0259-9$

NOGUEIRA, M.A.; CARDOSO, E.J.B.N. Colonização radicular e produção de micélio externo por duas espécies de fungos micorrízicos arbusculares em soja. Revista Brasileira de Ciência do Solo, v.24, p.329-338, 2000.

PAULA, M.A.; SIQUEIRA, J.O.; HOSHIKA, E. Crescimento, nutrição e produçấo de soja inoculada com populaçóes de fungos micorrízicos vesículo-arbusculares. Revista Brasileira de Ciência do Solo, v.14, p.151-156, 1990.

PESSOA, A.C.S.; ANTONIOLLI, Z.I.; DELLA-JUSTINA, M.E.; FIGUEIREDO, L.G.B. Fungos micorrízicos nativos e Glomus clarum no rendimento de trevo vesiculoso cultivado em condiçôes naturais e modificadas pela calagem e aplicação de fósforo. Ciência Rural, v.27, p.61-66, 1997.

PHILLIPS, J.M.; HAYMAN, D.S. Improved procedures for clearing roots and staining parasitic and vesicular-arbuscular mycorrhizal fungi for rapid assessment of infection. Transactions of the British Mycological Society, v.55, p.158-161, 1970. DOI: 10.1016/S0007-1536(70)80110-3

PLENCHETTE, C.; FORTIN, J.A.; FURLAN, V. Growth responses of several plant species to mycorrhizae in a soil of moderate P-fertility. Plant and Soil, v.70, p.199-209, 1983. DOI: 10.1007/BF02374780

PROCÓPIO, S.O.; SANTOS, J.B.; PIRES, F.R.; SILVA, A.A.; MENDONÇA, E.S. Absorção e utilização do fósforo pelas culturas da soja e do feijão e por plantas daninhas. Revista Brasileira de Ciência do Solo, v.29, p.911-921, 2005. DOI: 10.1590/S010006832005000600009

QUAGGIO, J.A.; GODOY, I.J. Amendoim. In: RAIJ, B. van; CANTARELLA, H.; QUAGGIO, J.A.; FURLANI, A.M.C. Recomendaçóes de Adubação e Calagem para o Estado de São Paulo. Campinas: IAC, 1996. 192p. (Boletim Técnico 100) 
ROCHA, F.S.; SAGGIN JÚNIOR, O.J.; SILVA, E.M.R.; LIMA, W.L. Dependência e resposta de mudas de cedro a fungos micorrízicos arbusculares. Pesquisa Agropecuária Brasileira, v.41, p.77-84, 2006. DOI: 10.1590/S0100-204X2006000100011

SENA, J.O.A.; LABATE, C.A.; CARDOSO, E.J.B.N. Caracterização fisológica da reduçáo de crescimento de mudas de citros micorrizadas em altas doses de fósforo. Revista Brasileira de Ciência do Solo, v.28, p.827-832, 2004. DOI: 10.1590/S0100-06832004000500005

SIDDIQI, M.Y.; GLASS, A.D.M. Utilization index: a modified approach to the estimation and comparison of nutrients utilization efficiency in plants. Journal of Plant Nutrition, v.4, p.286-302, 1981. DOI: $10.1080 / 01904168109362919$

SILVEIRA, A.P.D.; CARDOSO, E.J.B.N. Arbuscular mycorrhiza and kinetic parameters of phosphorus absorption by bean plants.
Scientia Agricola, v.61, p.203-239, 2004. DOI: 10.1590/S010390162004000200013

SIQUEIRA, J.O.; COLOZZI-FILHO, A.; FARIA, F.H.S. Efetividade simbiótica de fungos micorrízicos vesículo-arbusculares para o algodoeiro. Revista Brasileira de Ciência do Solo, v.10, p.213-218, 1986.

SMITH, S.E.; READ, D.J. Mycorrhizal symbiosis. $3^{\text {rd }}$ Ed. California: Academic Press, 2008.

ZAMBROSI, F.C.B.; MATTOS JR., D.; FURLANI, P.R.; QUAGGIO, J.A.; BOARETTO, R.M. Eficiência de absorção e utilização de fósforo em porta-enxertos cítricos. Revista Brasileira de Ciência do Solo, v.36, p.485-496, 2012. DOI: 10.1590/S010006832012000200018 